\title{
RIGHT VENTRICULAR PRESSURE FLOW RELATIONSHIPS AND INTRACARDIAC VENTURI EFFECTS IN FALLOT'S TETRALOGY
}

\author{
BY \\ HAMISH WATSON, A. M. BRECKENRIDGE, AND K. G. LOWE \\ From the Department of Medicine in the University of St. Andrews, Queen's College, Dundee
}

Received March 5, 1964

Recent studies of pressure flow relationships in isolated pulmonary valvular stenosis have demonistrated that, broadly speaking, they conform to certain well-known physical laws (Watson et al., 1960; Watson and Lowe, 1962), and a better understanding of right ventricular function in that condition has prompted us to make a similar study in patients with Fallot's tetralogy.

Since there is still some disagreement about which cases should rightly be grouped under this title (Rosenblum et al., 1962; Coelho et al., 1961), we have considered only the so-called "classical" cases in our analysis: those with pulmonary stenosis, either valvular, infundibular, or both, and the characteristic ventricular septal defect that allows blood to pass freely from the right ventricle into an overriding aorta. When compared with those having a normal aortic root and an intact interventricular septum, it is obvious that the presence of this defect modifies the hæmodynamic effects of obstruction to the outflow of blood from the right side of the heart. Because of it, the pressure in the right ventricle can never rise above that in the left in its attempt to force blood into the lungs, since at this level right-to-left shunting takes place and blood escapes into the systemic circulation. This affects the form as well as the height of the right ventricular pressure pulse and accounts for the difference between the peaked main cavity pulse waves of severe isolated pulmonary stenosis (Fineberg and Wiggers, 1936) and the flat topped ones so often seen in Fallot's tetralogy.

The other differences between the two conditions are due to the great variety and combination of stenotic lesions that may be present between the tricuspid valve and the pulmonary trunk in the tetralogy.

\section{NATURE OF OBSTRUCTION}

A study of right ventricular pressure flow relations must take into account the types and sites of these obstructions, because such factors as the length/diameter ratio of the stenosis and the nature of the chamber distal to the constriction greatly influence the hæmodynamics of the blood flowing through it. It is not yet easy to determine the true relative incidence of the various types of pulmonary stenosis found in Fallot's tetralogy, since it has only been possible to make an accurate anatomical assessment during open heart surgery for a relatively short time. Before this, such information as was available came from post-mortem studies, and as Keith, Rowe, and Vlad (1958) have pointed out in their review of earlier reports, there is considerable variation from series to series. Their averages of 13 per cent valvular, 60 per cent infundibular, and 27 per cent combined stenoses do not include pulmonary atresia which, it is generally agreed, accounts for about 1 case in 5 , a figure that corresponds closely to Blalock's experience in a largely post-operative group (Johns, Williams, and Blalock, 1953). This high percentage of pulmonary atresia has, of course, been found amongst patients with the most severe Fallot's tetralogy who are least likely to surmount major 
surgical procedures or to survive for many years if left untreated, and gives a false impression of the true incidence of this type of obstruction, as do some early operative series in which almost half the cases were said to have valvular stenosis as an isolated lesion (Glover et al., 1952; Brinton and Campbell, 1953).

Whatever the truth of the matter, it is now generally agreed that some degree of infundibular obstruction, either alone or in combination with valvular stenosis, is present in the great majority of cases. This may be situated high, mid, or low in the outflow tract, vary greatly in length and diameter, and be fixed and fibrous, or muscular and contractile. Despite improved methods of diagnosis (Watson et al., 1958, 1960; Watson and Pickard, 1964), it is still often difficult to assess its exact nature and severity, and for this reason we have endeavoured to make a more sophisticated study of the information obtained during cardiac catheterization in the hope that correlation of the various pressure pulse patterns with simultaneously recorded intracardiac electrograms will enable us to build up a much more accurate picture of the outflow tract than has previously been possible.

Before describing them in some detail, we would like to re-emphasize a few of the difficulties that may be encountered during their recording and subsequent analysis. It is not always possible to catheterize the pulmonary artery in Fallot's tetralogy and even when this has been accomplished, unspoiled records are difficult to obtain. The slowest and most careful withdrawal from pulmonary trunk to right ventricle may not succeed in preventing the catheter from passing through the infundibulum between two ventricular beats without recording a single electrocardiographic complex or pressure pulse. When they are recorded, the infundibular pressure pulse waves are often grossly distorted by ventricular arrhythmias, and pressure artefacts are commonly seen as the catheter tip passes down through the right ventricular outflow tract. We have found a simultaneously recorded intracardiac electrogram particularly helpful in detecting artefacts in pressure pulse tracings (Watson, 1964), since any ST elevations in this lead indicate that the electrode at the tip of the catheter has been in contact with the endocardium, and all pulse waves recorded in their presence should be viewed with suspicion. Those seen in the outflow tract of the right ventricle in Fig. 1, for example, are curiously broadened when compared with those in the main cavity. Such widening has been thought by some to indicate delayed relaxation of the infundibular musculature, but a study of the intracardiac lead in this case showed that it was only present when the electrogram was grossly distorted by ventricular contact currents.

A similar phenomenon is seen in Fig. 2. This time the broad pressure pulses were recorded near the apex of the left ventricle and once again the ST shifts on the intracardiac electrogram indicate that the electrode at the tip of the catheter has been in contact with the heart wall. The left heart had been catheterized through the foramen ovale, and when the catheter was withdrawn towards the mitral valve the contact currents subsided and the left ventricular pressure pulses regained a normal wave form.

Such changes in the intracardiac electrogram do not necessarily mean that the pulse wave is distorted, because, as the electrode surrounds the catheter a millimeter or two from its tip, it may contact the heart wall when the end-hole is not obstructed (Watson, 1964, Fig. 10). When they are present, however, they should make one cautious about the interpretation of any other recorded data.

\section{MeTHODS AND MATERIALS}

All patients have been catheterized from the right groin using electrode catheters manufactured by the United States Catheter and Instrument Corporation. The illustrations were recorded during cardiac catheterization on 6 in. Kodak RP 30 paper using New Electronic Products photographic equipment at a paper speed of $25 \mathrm{~mm}$. a second. Each tracing shows a withdrawal from the pulmonary artery into the main cavity of the right ventricle with a standard scalar electrocardiographic lead II at the top, the electrocardiogram recorded inside the heart in the middle and the pressure pulse tracing at the bottom. The intracardiac electrocardiograms were standardized at 2 millivolts per centimeter deflection and have been emphasized with black ink where necessary to make them clearly visible. 


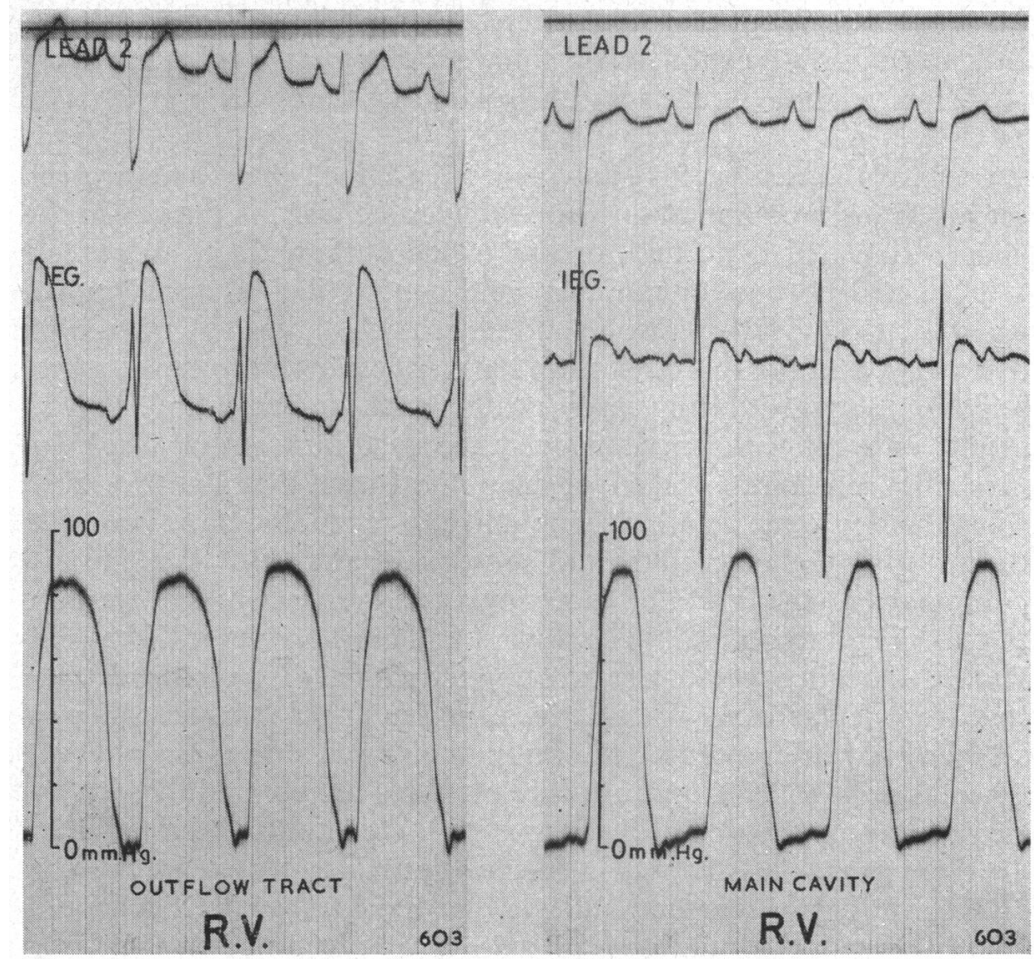

FIG. 1.-This illustrates the value of the intracardiac electrogram in detecting pressure artefacts. The curiously broadened pressure pulses are only present when gross ST elevations indicate contact between the electrode at the tip of the catheter and the infundibular endocardium. On withdrawal, contact is lost, and a normal wave form reappears in the cavity of the right ventricle.

\section{Pressure Pulse Patterns}

The pressure pulse patterns in Fallot's tetralogy vary greatly from case to case and depend on the nature and severity of the obstruction to the outflow of blood from the right ventricle. Where this is complete, there is of course no flow into the lungs, and for this reason pulmonary atresia need not be discussed further in the context of this paper. It should be appreciated, however, that many deeply cyanosed children with severe Fallot's tetralogy have what almost amounts to pulmonary atresia, and an example of the gross infundibular narrowing that may exist in these patients is illustrated on a lateral angiocardiogram in Fig. 3. This film, taken during diastole after a selective injection of contrast into the right ventricle has simultaneously opacified both great arterial trunks, shows that the whole length of the right ventricular outflow tract, from the main cavity of the ventricle to the dilated pulmonary trunk, is just barely patent.

Pressure flow studies in such cases are not easy, since it is often extremely difficult to find the tiny outlet from the right ventricular chamber during cardiac catheterization. Fig. 4 illustrates the type of withdrawal tracing that will be obtained if a catheter can be passed through it into the pulmonary artery. In these circumstances the pressure tracing alone will not localize the site of the stenosis, and it is impossible to tell, without the aid of the intracardiac lead, whether it is at the valve or in the infundibulum. A striking change in the amplitude of the electrogram, however, signals the passage of the electrode through the pulmonary valve and indicates that the large $\mathrm{rS}$ complexes that are seen on the tracing before the pressure gradient appears were recorded as the catheter tip passed down through the outflow tract distal to the stenosis. In this group of cases the systolic pressure gradient between the main cavity and infundibulum of the right ventricle is always large and as a rule there is 


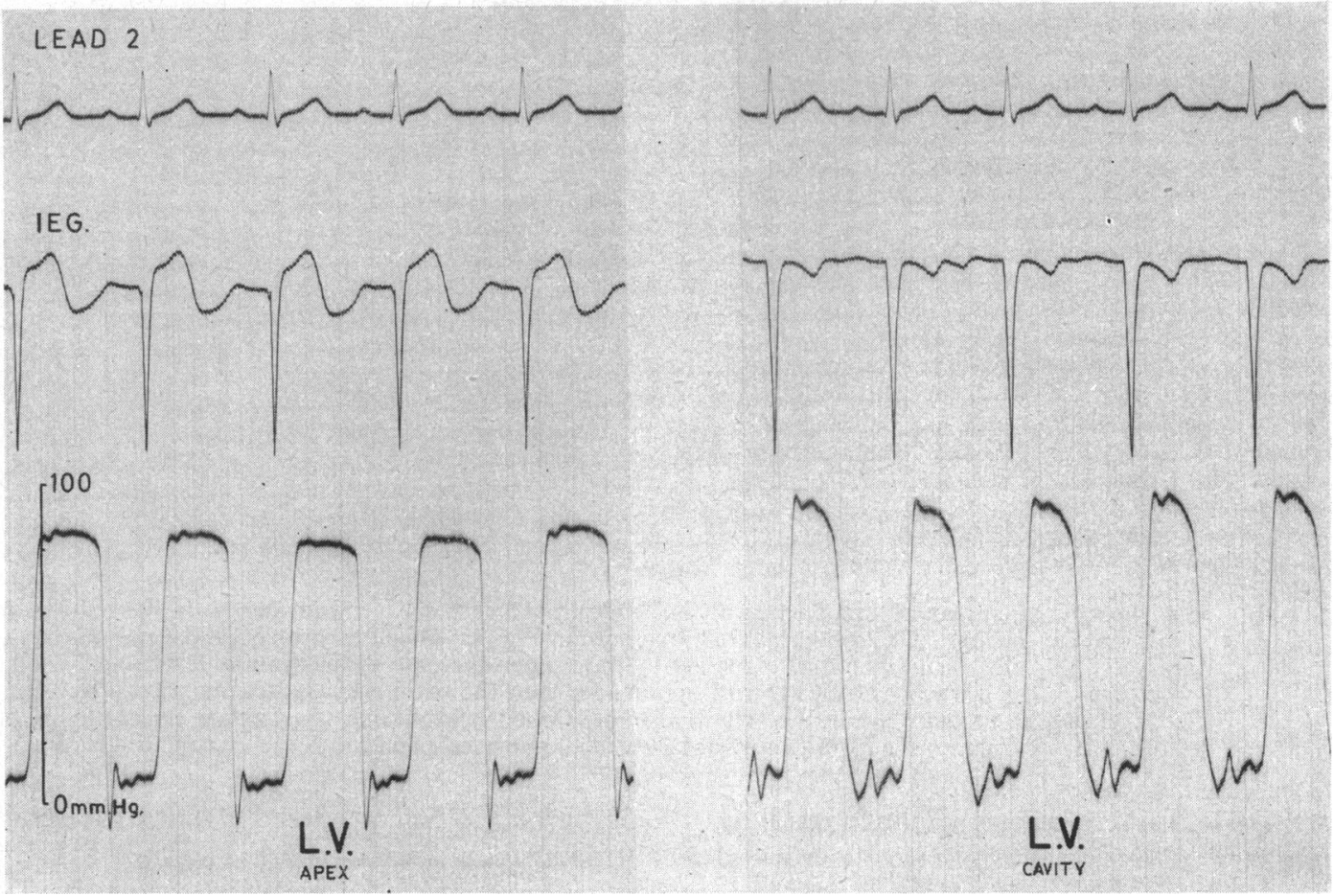

FIG. 2.-Distortion of the pressure pulses and elevation of the ST segments while an electrode-tipped catheter was in contact with the heart wall at the apex of the left ventricle. When the catheter was pulled back towards the mitral valve, the contact currents subsided and with the tip free in the cavity of the ventricle the pressure pulse regained its normal appearance.

little or no recognizable pressure pulse in either the outflow tract or the pulmonary trunk because there is virtually no infundibular musculature to restore a wave form to forward flow at the lowered pressure.

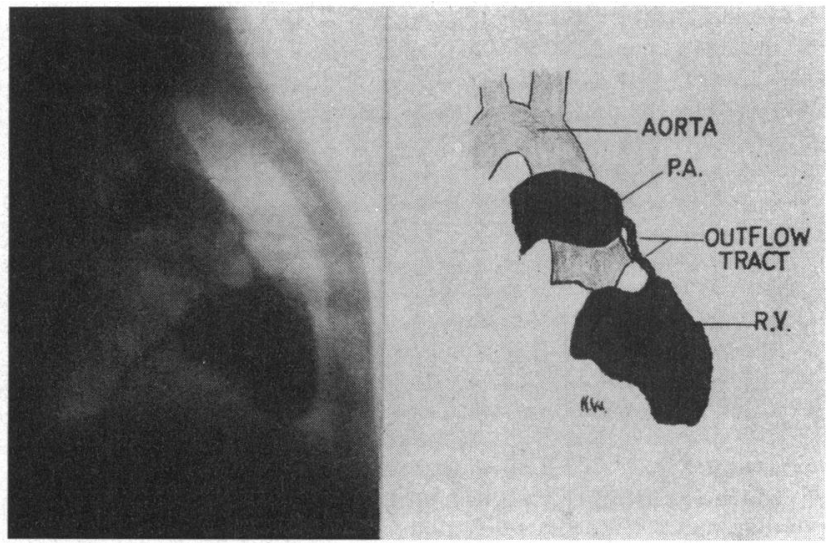

Fig. 3.-A still, excerpted from a right lateral cine-angiocardiogram taken at 50 frames a second, after an injection of 75 per cent Triosil into the right ventricular cavity in a case of severe Fallot's tetralogy, which shows gross narrowing throughout the entire length of the right ventricular outflow tract. 


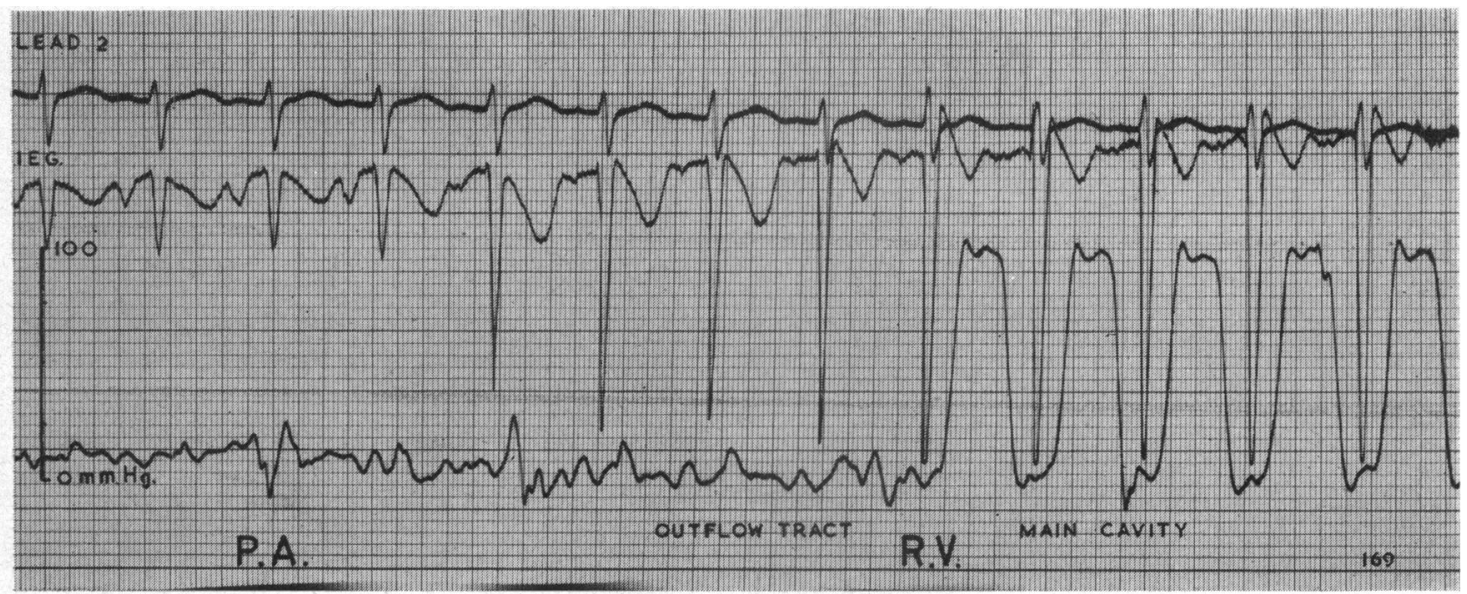

FIG. 4.-The type of tracing obtained when an electrode-tipped catheter is withdrawn from the pulmonary trunk through an outflow tract like that seen in Fig. 3. Its passage through the pulmonary valve into the infundibulum is signalled by the sudden increase in the amplitude of the $r S$ complexes on the intracardiac electrocardiogram, and into the main right ventricular cavity by the large systolic pressure gradient. The poorly developed infundibular muscles are unable to restore a recognizable wave form to forward flow at the lowered pressure.

Of the less severe cases of Fallot's tetralogy, the first group to be considered is that with isolated pulmonary valvular stenosis: Fig. 5 is a withdrawal tracing from such a case. The site of the pulmonary valve is again pinpointed by the intracardiac electrogram and a negative systolic pressure wave, or Venturi effect, is seen just before the catheter tip slips back through it into the heart. The pulse waves in the outflow tract, narrowed over its whole length by hypertrophy of the infundibular muscles, show the same striking narrowing when compared with those of the main cavity, as we have described (Watson and Lowe, 1962) in cases in which severe pulmonary valvular stenosis occurs as an isolated lesion with an intact aortic root. The only differences between withdrawal tracings in the two conditions are that in Fallot's tetralogy the main cavity pulse waves have a dis-

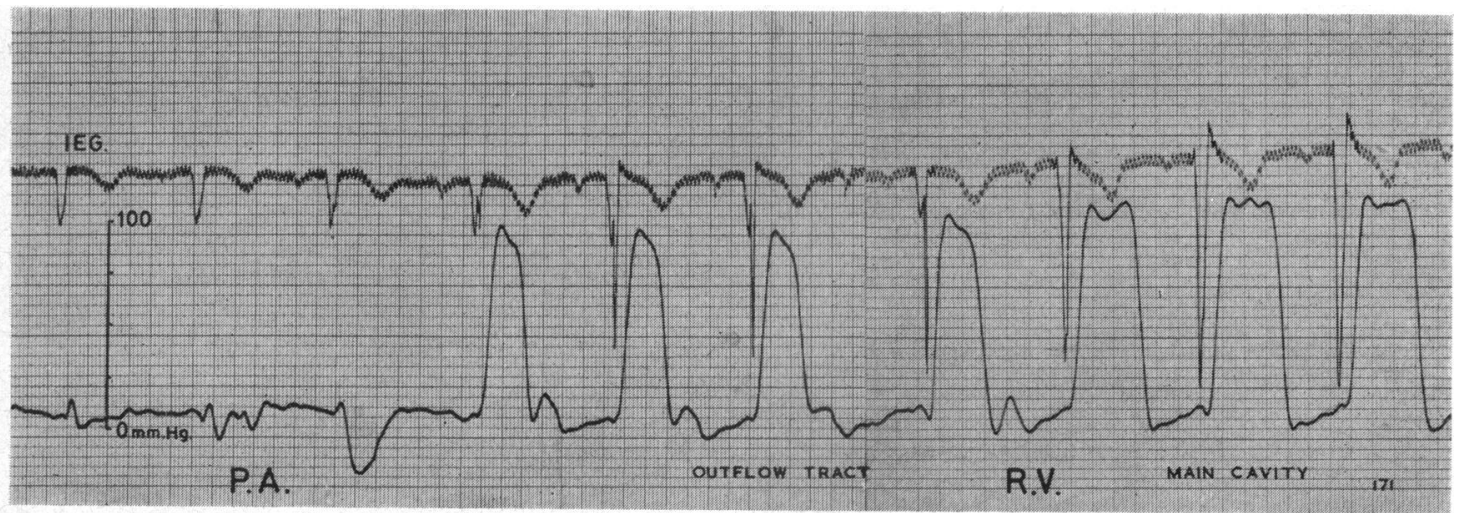

FIG. 5.-A withdrawal tracing from the pulmonary artery in a case of Fallot's tetralogy with isolated pulmonary valvular stenosis. The pulmonary valve is again pin-pointed by the intracardiac electrogram and a negative systolic pressure wave, or Venturi effect, is seen just before the catheter tip slips back through it into the lumen of the outflow tract which has been narrowed over its whole length by muscular hypertrophy. Blood flowing through it with increasing velocity causes a late systolic pressure drop in the pulse wave which does not regain its normal width and characteristic plateau top until the main cavity of the right ventricle is reached. 


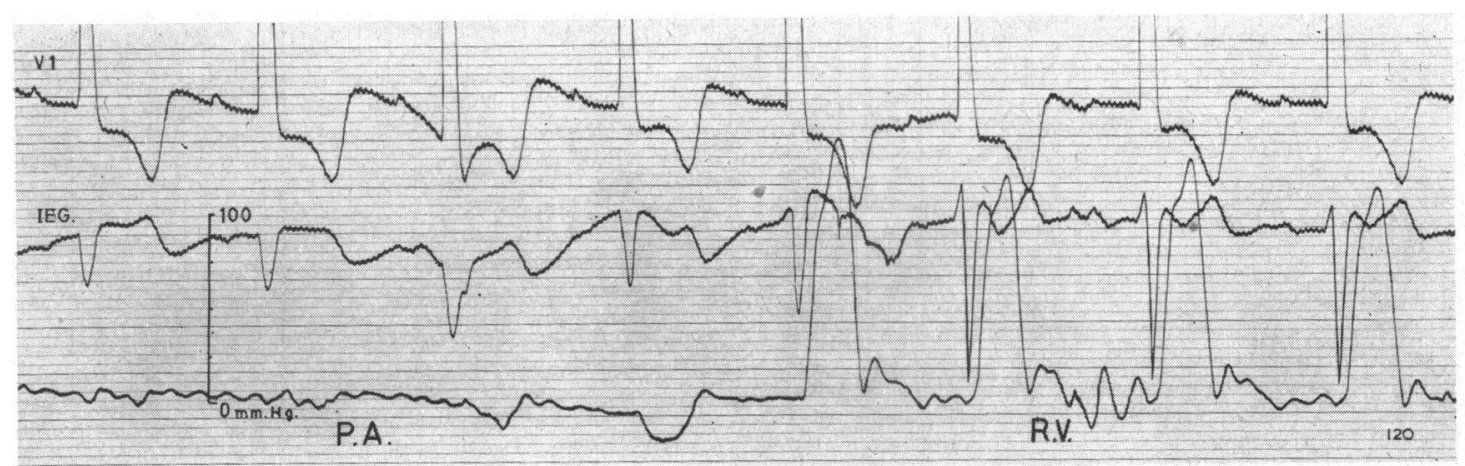

FIG. 6.-Pressure pulses similar to those seen in Fig. 5 from a case where a thin muscular diaphragm obstructed the outflow tract about $5 \mathrm{~mm}$. below an apparently healthy pulmonary valve. The negative systolic pressure pulse is a true intracardiac Venturi effect and a similar fall in pressure will be seen at the site of short length/diameter ratio obstructions at any level in the infundibulum.

tinctive plateau top and their maximum systolic pressure is limited to systemic level. In each, obstruction to the flow of blood from the right ventricle into the lungs has a similar effect on the pressure pulse patterns. A Venturi effect will be present at the stenosed pulmonary valve where the length/diameter ratio of the obstruction is small, and a late systolic pressure drop due to viscous resistance to blood flow will be seen in the outflow tract where the ratio is large.

When considering the effects of isolated infundibular stenosis on pressure pulse patterns much will depend on the site as well as the nature of the obstruction. A high infundibular obstruction with a short length/diameter ratio, for example, will produce hæmodynamic effects that are virtually indistinguishable from those of valvular stenosis. This is demonstrated in Fig. 6 which was recorded during the pre-operative investigation of a girl with pulmonary stenosis and a secundum type atrial septal defect, who was thought clinically to have the tetralogy of Fallot. Though the withdrawal tracing from the pulmonary trunk shows negative systolic pressure waves just before the gradient, and the pulse waves in the outflow tract are also typical of those seen in valvular lesions, this patient did not have pulmonary valvular stenosis. At operation, a thin muscular diaphragm was found to be obstructing the outflow tract about $5 \mathrm{~mm}$. below the valve. The fall in pressure that occurs as the blood flows through it with increased velocity is a true intracardiac Venturi effect, and similar phenomena will be seen in the presence of short length/diameter ratio obstructions at any level in the infundibulum.

The form of the pressure pulse distal to the infundibular stenosis depends to a large extent on the nature of the infundibular muscle surrounding the outflow tract distal to the stenosis. In its most severe form (Fig. 4), where a low infundibular obstruction is as it were continuous with a long narrow fibrous and rudimentary outflow tract, there is practically no pulsation. At the opposite end of the scale, where a low infundibular obstruction has converted the outflow tract into a third ventricle, pulsation is essentially normal when the catheter tip is clear of the obstruction. An example from a case of this type is illustrated in Fig. 7. The sudden change in the intracardiac electrogram on this tracing locates the pulmonary valve, and confirms that there is no valvular stenosis, while the systolic pressure gradient marks the site of the obstruction low in the infundibulum. Three pulse waves showing intracardiac Venturi effects are seen as the catheter tip passes back through it into the main cavity of the right ventricle. The fall in pressure during systole is short lived in such cases of isolated infundibular stenosis, because distal to the obstruction the lumen of the outflow tract is of reasonable size and the muscles encircling it are able to restore a normal ventricular wave form, which is then propagated into the pulmonary arteries.

This is in sharp contradistinction to the pattern seen where there is pulmonary valvular stenosis as well. In these circumstances, hypertrophy of the infundibular muscles behind this stenosis 
LEAD 2

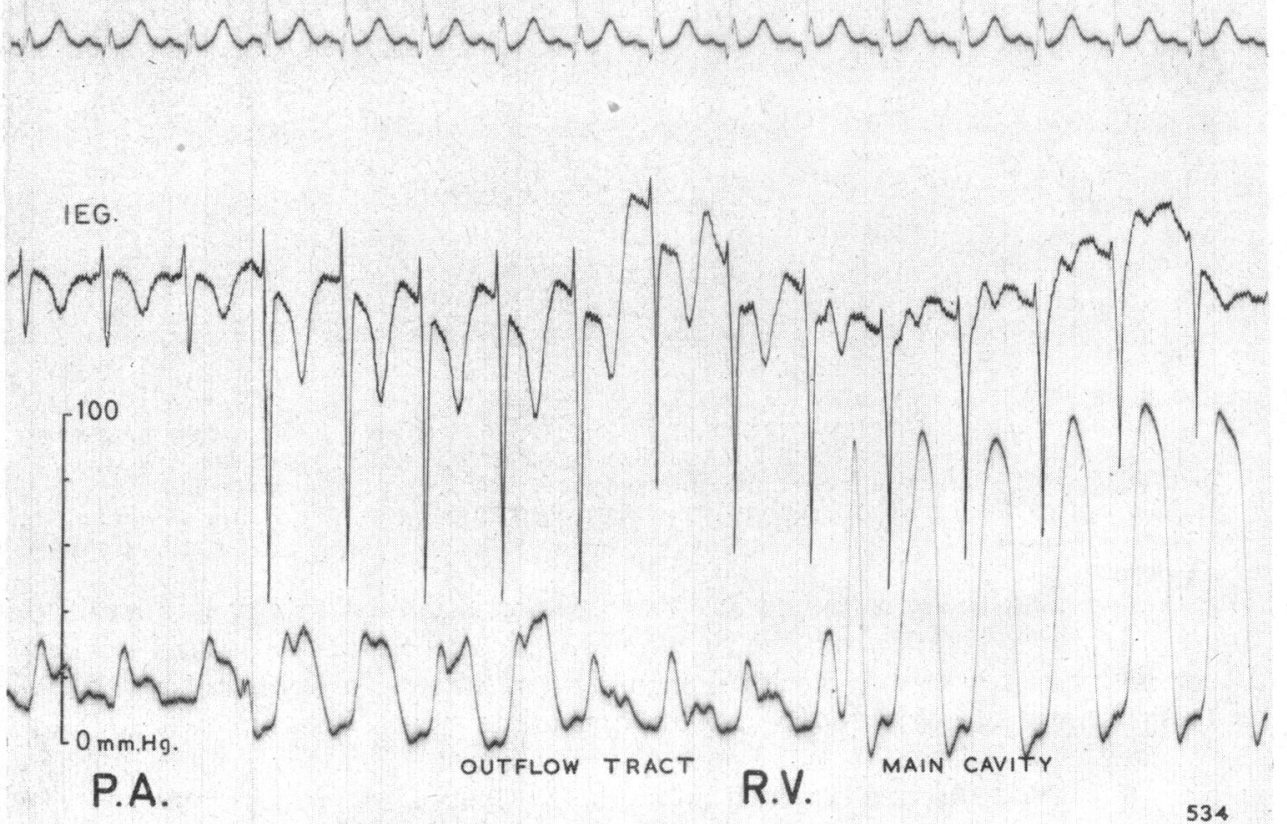

FIG. 7.-A record from a case of Fallot's tetralogy where a low infundibular obstruction has converted the right ventricular outflow tract into a "third ventricle". The intracardiac electrogram confirms the absence of pulmonary valvular stenosis and three pulse waves showing intracardiac Venturi effects are seen just before the systolic pressure gradient appears on the tracing. Distal to this localized stenosis, the outflow tract has a wide lumen and the infundibular muscles restore a normal ventricular wave form to the blood flowing through it.

reduces the lumen of the outflow tract, and the fall in pressure that occurs during systole is present over its entire length. Initiated by a Venturi effect, the pressure fall at the site of infundibular obstruction is maintained by viscous resistance as the infundibular muscles contract to force blood through the stenosed valve. These effects are demonstrated in Fig. 8 on a withdrawal tracing recorded during the pre-operative investigation of a case of Fallot's tetralogy with combined valvular and infundibular lesions. The sites of the stenoses are indicated by the systolic pressure gradients and the intracardiac electrogram confirms that the pressure pulse waves between them were recorded as the catheter tip moved down the outflow tract. As in Fig. 7, its passage through the infundibular stenosis is marked by a transitional pressure wave, but thereafter the pulse never regains a normal wave form because muscular hypertrophy has narrowed the lumen over its entire length, and the late systolic pressure drop persists even when well clear of the infundibular obstruction.

These then are the basic pressure pulse patterns seen in the right ventricular outflow tract in Fallot's tetralogy. As one might expect in a condition with such varied pathology, all possible combinations and modifications of them are seen in day-to-day practice. In Fig. 9, for example, there are a great variety of pressure pulse waves on a record made as a catheter was pulled back from the pulmonary artery into the main cavity of the right ventricle in a case with combined valvular and infundibular lesions. Its passage through an obstruction low in the outflow tract has unfortunately caused an ectopic beat at the point where an intracardiac Venturi effect might have been recorded, but the rapidity with which a ventricular pulse having a relatively normal wave form reappears suggests a lesion with a short length/diameter ratio leading into a "third ventricle" type of outflow tract. Approaching the stenosed pulmonary valve, however, an area of secondary muscular 


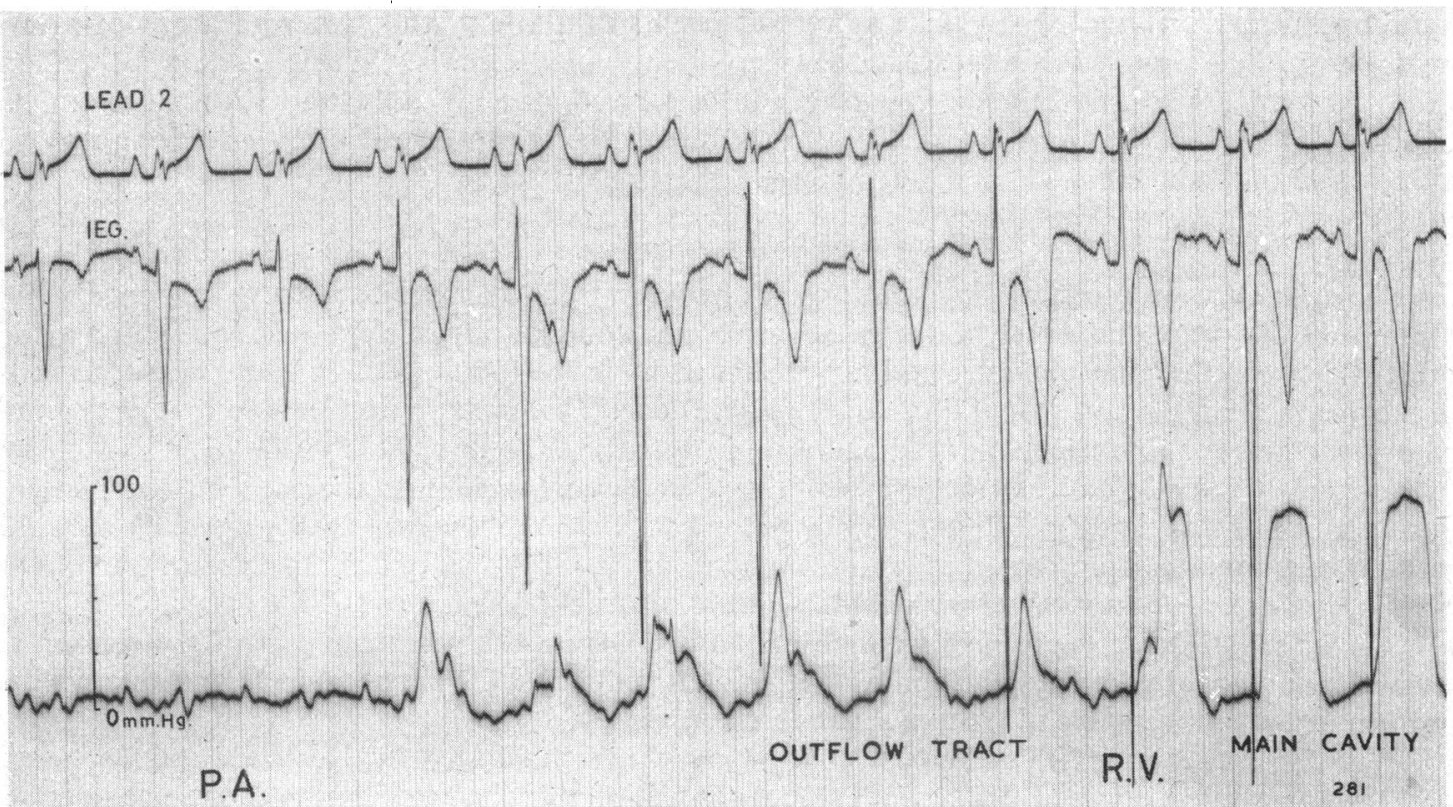

FIG. 8.-A record from a patient with combined valvular and infundibular stenosis. The intracardiac electrogram confirms that the pressure pulses between the systolic gradients were recorded as the catheter tip moved down through the infundibulum. The fall in pressure during systole initiated at the site of the infundibular stenosis is maintained throughout the entire length of the outflow tract, because its lumen has been narrowed by hypertrophy of the muscles behind the valvular obstruction. In such cases the infundibular pressure pulse never regains a normal wave form.

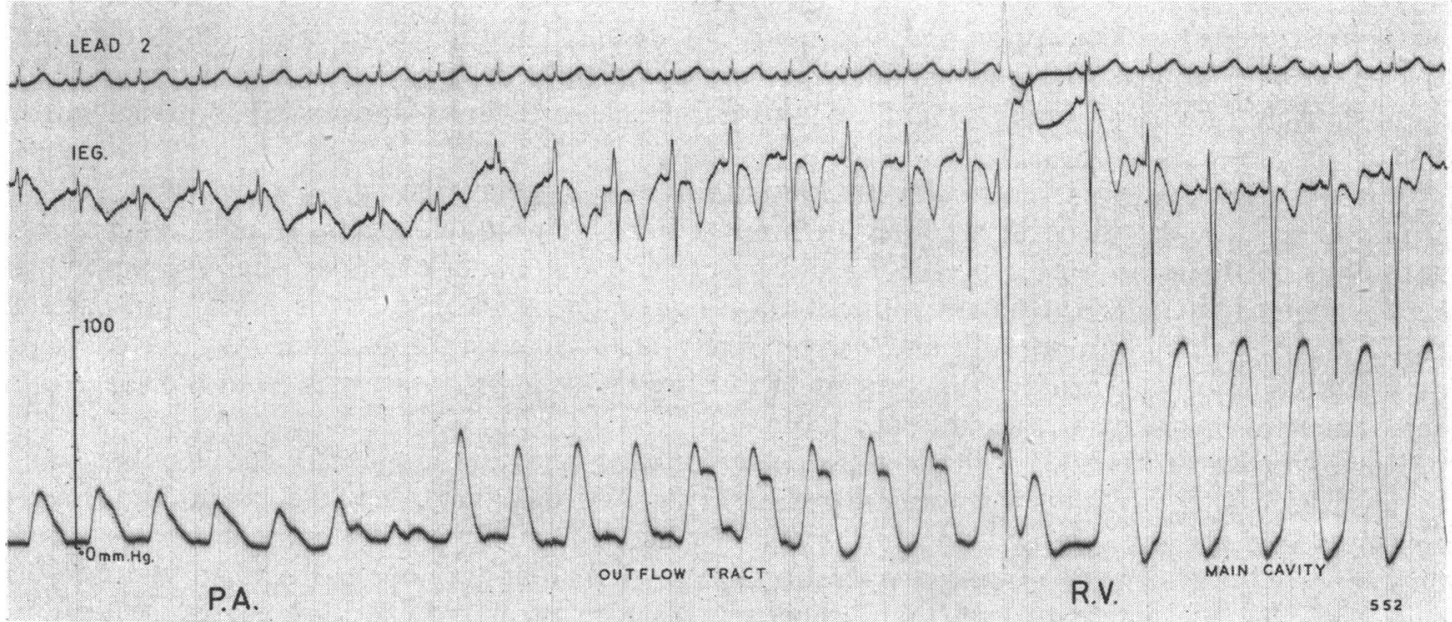

FIG. 9.-The variety of pressure pulses that may be recorded during withdrawal from the pulmonary artery into the right ventricle in Fallot's tetralogy with combined valvular and infundibular stenosis. A systolic gradient marks the site of infundibular stenosis and an ectopic beat spoils the record where an intracardiac Venturi effect might have been recorded as velocity increased to maintain flow through the obstruction. Thereafter, a well developed infundibulum restores a normal wave form to the pressure pulse for a few beats before an area of secondary muscular hypertrophy once more narrows its lumen. This results in a further fall in pressure during systole until a systolic pressure gradient and Venturi effect appear at the stenosed pulmonary valve. 
hypertrophy narrows the lumen once more and results in a late systolic pressure drop for a few beats before the Venturi effect appears at the site of the valvular stenosis. We do not suggest that an exact anatomical picture of the outflow tract can be arrived at in all cases, but that a more critical appraisal of the information recorded during cardiac catheterization will often go a long way to doing so in many of them.

\section{Discussion}

The differences in pressure flow relationships between isolated pulmonary valvular stenosis and Fallot's tetralogy are due largely to the great variety and combination of stenotic lesions that may be present between the tricuspid valve and pulmonary trunk in the tetralogy of Fallot. These, according to Keith (1909), result from failure of the bulbus cordis to undergo normal development in the embryonic heart. His theory that the bulbus is a transitory structure, which soon loses its identity by being absorbed into the ventricular part of the heart, has coloured most thoughts on such matters during much of this century. That it largely becomes the infundibulum of the right ventricle would certainly explain the combination of lesions found in the tetralogy, since defective absorption of the bulbus would account not only for the outflow tract obstruction, but also for the particular type of ventricular septal defect seen in this condition. For whereas the muscular part of the septum is formed by outgrowths of the developing right and left ventricles, the membraneous portion appears to form in the bulbus while it is still a separate chamber.

Recently, however, Keith's classical concept has been challenged by De Vries and Saunders (1962) who have re-examined Waterston's (1918) contention that the whole right ventricle has a common origin. This, too, is an attractive theory which might explain why the muscle of both the outflow tract and the main cavity of this chamber hypertrophy in response to severe valvular stenosis. It might also account for those rare cases of defective development where the whole right ventricular chamber, from tricuspid to pulmonary valves, is rudimentary and stenosed (Taussig, 1960). Whatever the merits of the respective arguments about the role of the bulbus cordis, and there are even those who deny its existence in the human heart (Dankmeijer, 1963), it is impossible to study the wide variety of obstructive lesions found in the tetralogy of Fallot without being impressed by the concept that defective absorption and fibrosis of the mesenchymatous tissue in the developing outflow tract could lead to all grades of stenoses from complete pulmonary atresia to division of the right ventricle into two almost separate chambers (Duckworth and Marquis, 1952; Duckworth, 1958).

When considering right ventricular function in Fallot's tetralogy, the possible importance of the infundibular musculature soon becomes apparent. For though phylogenetic studies indicate that the bulbus cordis is no longer present as a separate and functional chamber after the amphibia, there is increasing evidence that in some cases of congenital heart disease the septal and parietal muscle bands, which run spirally and crescentically around the outflow tract, may on occasion carry out a sort of rudimentary function by protecting the pulmonary arteries from the surge of a high ventricular pressure (Lynfield et al., 1961).

Reviewing their cases of Fallot's tetralogy, Rodbard and Shaffer (1956) and Rodbard and Rekate (1957) suggested that when the infundibular stenosis was due to a distorted and abnormally large muscle mass, the stenosed opening might be narrowed still further by muscular contraction. They reported a patient in whom, at operation, a muscular diaphragm was found to separate the main right ventricular cavity from its outflow tract, which increased the obstruction to the outflow of blood from the right ventricle by closing like a camera shutter during systole. Gasul and his colleagues (1957) reported that some infants, born with only large ventricular septal defects, developed a "high degree" of infundibular stenosis as a result of muscular hypertrophy and were thus transformed into cases resembling those with Fallot's tetralogy. Even where stenosis cannot be demonstrated at operation or necropsy (Watson and Lowe, 1965), there is ample evidence to support the concept that a physiological sphincter may exist in the absence of its anatomical exhibition ( $R$. Walmsley, 1963, personal communication). 
By suggesting purposeful action, such cases emphasize the complex nature of infundibular muscle function and suggest that physical forces as well as structural abnormalities may influence the evolution of congenital heart disease. As Nadas (1957) has pointed out, some anomalies although recognizable at birth do not assume their pathogenic characteristics until many months or years later: their natural history is partly determined by changes that take place in extrauterine life. Thus Taussig (1960) has recorded that the obstruction to the outflow of blood from the right ventricle in Fallot's tetralogy may become more severe because of fibro-elastotic thickening of the endocardium, calcium deposition, muscular hypertrophy, or simply because the abnormal infundibular tissues do not grow so fast as the other tissues. Rodbard (1956) has suggested that the infundibulum, by contracting during systole, may produce a powerful jet which may bring about progressive fibrosis and ultimate stenosis of the pulmonary valve leaflets. Recent observations by De Vries and Saunders (1962) on the interdependence of ontogenetic segmental growth potential and fluid mechanics confirm that hæmodynamic forces not only modify the developing heart but they do so from a very early intrauterine age. Their studies on junctioning streams, and the differing effects of spiral and lateralized flow on luminal surfaces, has shed new light on the growth of the outflow tract and the possible ætiology of the lesions found in those who are born with the tetralogy of Fallot.

In view of the complexity of these lesions it is not surprising that it has always been difficult to predict their exact nature with any degree of confidence from pre-operative investigations. What is perhaps surprising is that so few attempts have been made to do so. During the early days of diagnostic cardiac catheterization most investigators were satisfied with simple pressure gradients as evidence of the presence and location of stenosis and little attention was paid to the actual form of the pressure pulse waves till paradoxical negative systolic pressure waves, or Venturi effects, were described at the site of obstruction in isolated pulmonary valvular stenosis (Sobin et al., 1954; Bouchard and Cornu, 1954). Harris (1955) was one of the first to demonstrate that the right ventricular pressure pulse pattern varied with different lesions and, puzzled by the wave form in two cases of isolated infundibular stenosis, suggested that it might be explained by some variation in the resistance to the outflow of blood during systole. A mid-systolic dip in the outflow tract pressure pulse was described by Kjellberg et al. (1955) in one case of isolated infundibular stenosis, but no serious attempt to interpret the functional significance of the pressure pulse patterns in Fallot's tetralogy was made until Rodbard and Shaffer (1956) tried to differentiate between fixed fibrous and muscular contractile lesions by a detailed analysis of the infundibular pressure pulse waves. Their conclusions that velocity effects were unimportant were not confirmed in a similar study on patients with isolated pulmonary stenosis by Johnson (1959), who reported that contractile and fixed lesions could be differentiated by timing the infundibular contraction in relation to right ventricular systole. While agreeing with his contention that the early fall of pressure in the infundibulum is due to increased velocity of flow through the obstruction, we have not as yet seen his phenomenon of infundibular contraction continuing after right ventricular contraction has ceased.

Impressed by the narrow lumen of the infundibulum in cine-angiocardiographic studies of severe isolated pulmonary valvular steosis, Watson and Lowe (1962) showed that the fall in pressure during systole, resulting from forward flow at increased velocity and revealed by a Venturi effect at the valve, was matched by a similar fall in pressure during systole in the outflow tract. They found that, though complex, the pressure flow relationships were governed by such well-known principles as Poiseuille's Law and the Bernoulli theorem, and depended to a large degree on the length/diameter ratio of the obstruction. Where this was small, as in valvular stenosis, or, as has been demonstrated in this paper, at the site of a localized infundibular obstruction, a transient Venturi effect might be recorded; where it was large, as in an infundibulum narrowed over its whole length by muscular hypertrophy, a sustained fall in pressure due largely to viscous resistance to fluid flow could be expected.

The present analysis has shown that the same physical laws are responsible for the great variety of pressure pulse patterns seen during catheter withdrawal through the right ventricular outflow tract in Fallot's tetralogy. Clues to the exact nature of the many types of obstructive lesions that 
may be present in this condition are provided both from pressure gradients and by careful study of the pressure pulse waves themselves. It should be remembered, however, that the magnitude of the gradients may be misleading, since there is commonly more than one, and a fall in pressure at a site of stenosis is only of value in determining its severity when the flow rate across it is known. As in isolated pulmonary valvular stenosis, we have found that careful scrutiny of simultaneously recorded intracardiac electrograms and pressure pulses is of great value in the pre-operative investigation of Fallot's tetralogy. Such catheter data when correlated with biplane cine-angiocardiographic studies enable us to determine not only the anatomy of these various congenital and acquired lesions, but also to assess, with a fair degree of accuracy, their functional effect on the hæmodynamics of the outflow of blood from the right ventricular cavity.

\section{SUMMARY}

A better understanding of pressure flow relationships in isolated pulmonary valvular stenosis has prompted a similar study in Fallot's tetralogy.

An analysis of the various pressure pulse patterns and their correlation with simultaneously recorded intracardiac electrocardiograms has shown that outflow from the right side of the heart is influenced by the same physical laws in both conditions and has demonstrated that Venturi effects do occur inside the heart.

Structural abnormalities in the right ventricular outflow tract have been reviewed and the functional significance of the infundibulum has been considered in relation to its embryological origin.

The importance of fluid dynamics in determining the evolution of congenital heart lesions, in both intra- and extrauterine life, is stressed.

It is concluded that a more critical appraisal of catheter data will not only help to establish the anatomy of the various congenital and acquired infundibular lesions found in the tetralogy of Fallot, but will also go a long way to determine their effect on function. This information, when related to biplane cine-angiocardiographic studies, is of great value in the assessment of such patients with a view to surgical correction.

\section{REFERENCES}

Bouchard, F., and Cornu, C. (1954). Etude des courbes de pressions ventriculaire droite et artérielle pulmonaire dans les rétrécissements pulmonaires. Arch. Mal. Caur., 47, 417.

Brinton, W. D., and Campbell, M. (1953). Necropsies in some congenital diseases of the heart, mainly Fallot's tetralogy. Brit. Heart J., 15, 335.

Coelho, E., de Paiva, E., de Pádua, F., Nunes, A., Amram, S., e Sá, B., and Luis, S. (1961). Tetralogy of Fallot: Angiocardiographic, electrocardiographic, vectorcardiographic, and hemodynamic studies of the Fallot-type complex. Amer. J. Cardiol., 7, 538.

Dankmeijer, J. (1963). Cardiac malformations studied in relation to the embryonic development of the human heart. Boerhaave Course, Leiden, 24-27 April.

De Vries, P. A., and Saunders, J. B. de C. M. (1962). Development of the ventricles and spiral outflow tract in the human heart. Contr. Embryol. Instn., Carneg., 37, 87. No. 256.

Duckworth, J. W. A. (1958). Embryology of congenital heart disease. In Heart Disease in Infancy and Childhood, by J. D. Keith, R. D. Rowe, and P. Vlad, p. 106 Macmillan, New York.

- , and Marquis, R. M. (1952). The anatomy of pulmonary stenosis. In Proceedings of the British Cardiac Society. Brit. Heart J., 14, 546.

Fineberg, M. H., and Wiggers, C. J. (1936). Compensation and failure of the right ventricle. Amer. Heart J., $11,255$.

Gasul, B. M., Dillon, R. F., Vrla, V., and Hait, G. (1957). Ventricular septal defects; their natural transformation into those with infundibular stenosis or into the cyanotic or noncyanotic type of tetralogy of Fallot. J.Amer. med. Ass., 164, 847.

Glover, R. P., Bailey, C. P., O’Neill, T. J. E., Downing, D. F., and Wells, C. R. E. (1952). The direct intracardiac relief of pulmonary stenosis in the tetralogy of Fallot. J. thorac. Surg., 23, 14

Harris, P. (1955). Some variations in the shape of the pressure curve in the human right ventricle. Brit. Heart J., $17,173$.

Johns, T. N. P., Williams, G. R., and Blalock, A. (1953). The anatomy of pulmonary stenosis and atresia with comments on surgical therapy. Surgery, 33, 161.

Johnson, A. M. (1959). Functional infundibular stenosis: its differentiation from structural stenosis and its importance in atrial septal defect. Guy's Hosp. Rep., 108, 373.

Keith, A. (1909). The Hunterian Lectures on malformations of the heart. Lancet, 2, 359, 433, and 519. 
Keith, J. D., Rowe, R. D., and Vlad, P. (1958). Heart Disease in Infancy and Childhood. Macmillan, New York. Kjellberg, S. R., Mannheimer, E., Rudhe, U., and Jonsson, B. (1955). Diagnosis of Congenital Heart Disease. Year Book Publishers, Chicago.

Lynfield, J., Gasul, B. M., Arcilla, R., and Luan, L. L. (1961). The natural history of ventricular septal defects in infancy and childhood. Amer. J. Med., 30, 357

Nadas, A. S. (1957). Pediatric Cardiology. Saunders, Philadelphia.

Rodbard, S. (1956). Vascular modifications induced by flow. Amer. Heart J., 51, 926.

- and Rekate, A. C. (1957). Direct evidence of supraventricular sphincter action as a mechanism of pulmonic stenosis. Exp. Med. Surg., 15, 317.

—-, and Shaffer, A. B. (1956). Muscular contraction in the infundibular region as a mechanism of pulmonic stenosis in man. Amer. Heart J., 51, 885.

Rosenblum, R., Mark, H., Escher, D. J. W., Stern, W. Z., and Young, D. (1962). The differentiation of pulmonic stenosis, ventricular septal defect with normal aortic root from tetralogy of Fallot. Amer. Heart J., $64,746$.

Sobin, S. S., Carson, M. J., Johnson, J. L., and Baker, C. R. (1954). Pulmonary valvular stenosis with intact ventricular septum. Amer. Heart J., 48, 416.

Taussig, H. B. (1960). Congenital Malformations of the Heart, Vol. II, 2nd ed. Harvard University Press, Cambridge, Massachusetts.

Waterston, D. (1918). The development of the heart in man. Trans. roy. Soc. Edinb., 52 (part II), 257.

Watson, H. (1964). Electrode catheters and the diagnostic application of intracardiac electrography in small children. Circulation, 29, 284.

- , and Lowe, K. G. (1962). Ventricular pressure flow relationships in isolated pulmonary valvular stenosis. Brit. Heart J., 24, 431.

- and - (1965). Functional adaptations of the right ventricular outflow tract in congenital heart disease. Brit. Heart J. In the press.

—, and Pickard, C. (1964). Right and left heart biplane cine-angiocardiography. Brit. Heart J., $26,755$.

-_, L Lowe, K. G., and Hill, I. (1958). Selective cine-angiocardiography with image intensification. Brit. Heart J., 20, 459.

$-,-\longrightarrow, \frac{}{\longrightarrow}$, and $(1960)$. Cine-angiocardiographic studies of the outflow tract in isolated pulmonary valvular stenosis. Brit. Heart J., 22, 706. 